\title{
Electrical control of spin dynamics in finite one-dimensional systems
}

\author{
A. Pertsova, M. Stamenova, and S. Sanvito \\ School of Physics and CRANN, Trinity College, Dublin 2, Ireland \\ (Received 21 June 2011; revised manuscript received 23 September 2011; published 19 October 2011)
}

\begin{abstract}
We investigate the possibility of the electrical control of spin transfer in monoatomic chains incorporating spin impurities. Our theoretical framework is the mixed quantum-classical (Ehrenfest) description of the spin dynamics, in the spirit of the $s-d$ model, where the itinerant electrons are described by a tight-binding model while localized spins are treated classically. Our main focus is on the dynamical exchange interaction between two well-separated spins. This can be quantified by the transfer of excitations in the form of transverse spin oscillations. We systematically study the effect of an electrostatic gate bias $V_{g}$ on the interconnecting channel and we map out the long-range dynamical spin transfer as a function of $V_{g}$. We identify regions of $V_{g}$ giving rise to significant amplification of the spin transmission at low frequencies and relate this to the electronic structure of the channel.
\end{abstract}

DOI: 10.1103/PhysRevB.84.155436

PACS number(s): 75.78.-n, 75.30.Hx, 73.63.-b, 85.75.-d

\section{INTRODUCTION}

The rapid development of the field of spintronics ${ }^{1}$ over the past two decades has uncovered exciting and novel phenomena related to the dynamics of the electronic spin in a wide variety of systems, ranging from bulk materials to spatially confined structures. ${ }^{2}$ Fueled by the ever-growing needs for speed, capacity, and energy efficiency in computing, the central objective in understanding and ultimately controlling the spin properties in the solid state has been constantly shifting toward the nanoscale. At these lengths and times the conventional methods for spin control, based on magnetic fields alone, are limited by scalability issues. Alternative approaches are thus sought and they typically involve electric fields of some form. ${ }^{3}$

One way to manipulate spins by purely electrical means relies on the spin-transfer torque mechanism. ${ }^{4}$ This approach uses spin-polarized currents to control the direction of the magnetization and has been realized in various nanostructured materials ranging from magnetic multilayers ${ }^{5}$ to, more recently, single atoms in STM-type geometries. ${ }^{6}$ Alternative to electric current control is the optical control, such as in the laser-driven ultrafast magnetization switching. ${ }^{7,8}$ In a somewhat different context, the optical manipulation of single spins in bulk media ${ }^{9}$ is at the heart of the most promising candidates for the quantum information processing technology. ${ }^{10,11}$

Another alternative is based on the idea of an electrostatic control of the spin density, i.e., of the construction of spin-transistor type devices. ${ }^{12}$ Recently, the concept of gate-modulated spin-pumping ${ }^{13}$ transistors has been studied theoretically in infinite graphene strips with patterned magnetic implantations. ${ }^{14}$ Importantly, such devices rely on the efficient transport of spin information between two points in space and time, and require the possibility to actively tune the propagating spin signal during its transport. In this work we explore this possibility for atomistic spin conductors. We consider a finite monoatomic wire linking two localized spin-carrying impurities. When one of the localized spins is set into precession, it generates a perturbation in the spin density. This perturbation is carried through the wire by conduction electrons and can be detected in the dynamical response of the second spin. We show that the propagation of the spin signal and consequently the dynamical communication between the two spin centers can be tuned by means of an electrostatic gate applied to the interconnecting wire. Our main finding is an enhancement of the communication for a certain range of gate voltages. This is linked to the modification of the electronic structure of the wire induced by the applied electrostatic gate.

The theoretical framework of our method is the ab initio spin dynamics (SD) model introduced by Antropov et al. ${ }^{15}$ This rests on an adiabatic approximation which separates the spin degrees of freedom in metallic materials containing atoms with localized magnetic moments. Because of the very different characteristic energies of the itinerant and the localized spins, the two spin species can be modeled by separate albeit coupled equations of motion. The directions of the localized spins are the slow component compared to the itinerant spins because the intersite exchange integrals are typically much smaller than characteristic band energies. It has been demonstrated in Ref. 15 that a set of vectors representing the directions of the local spins can be introduced as collective variables within the standard local spin density approximation (LSDA). These vectors are treated as classical degrees of freedom, analogously to the nuclear coordinates in Born-Oppenheimer (BO) molecular dynamics.

Because of their considerable size (at least hundreds of atoms) the systems of interest for spintronics applications are still beyond the present numerical capabilities of the first-principles spin dynamics. ${ }^{15-18}$ They are often described by model Hamiltonians and treated within the linear response approximation. ${ }^{14}$ In this paper we adopt Antropov's quantumclassical SD model, but we treat it at the level of the Ehrenfest approximation ${ }^{19}$ instead of the $\mathrm{BO}$ approximation. This allows us to describe coherent effects in the motion of the local moments that would be lost within the $\mathrm{BO}$ approximation as they depend on finite coupling at frequencies characteristic of the electronic scale $(\mathrm{PHz})$.

We perform a fully microscopic description based on the time-dependent Schrödinger equation, which allows us to model the effects of arbitrary excitations. In other words, our simulations are not limited to small gate voltages, spatially continuous electrostatic potentials, or small-angle spin precession and long-wavelength spin waves. We use 
a single-band tight-binding (TB) Hamiltonian to model the itinerant $s$ electrons in our metallic wires and include local Heisenberg interaction with a number of magnetic ions (one or two in our model systems). The directions of the latter are modeled as classical angular momenta coupled to the instantaneous spin density at their site and they obey classical Hamiltonian equations of motion.

Since the existence of the localized spins is an essential requirement for the coupled quantum-classical SD, our model is most suitable for systems containing magnetic impurities embedded in nonmagnetic hosts. Building such magnetic nanostructures atom-by-atom has now become possible and a number of relevant experimental setups, including noble metal surfaces with adsorbed transition metal atoms ${ }^{20}$ and individual magnetic adatoms or atomic chains on insulating surfaces, ${ }^{21}$ can be found in the literature. Note that an extension of the $s$ - $d$ model to quantum local spins has been recently proved very successful in describing inelastic spin excitations in scanning tunnel spectroscopy experiments for magnetic adatoms on surfaces. $^{22}$

Although our model can be easily extended to higher dimensions, here we have focused on spin dynamics in a one-dimensional structure. A possible realization of such a model system are metallic carbon nanotubes doped with single substitutional magnetic impurities, which have been recently proposed as promising candidates for spintronics applications. $^{23}$ In a broader context, our model system is as a schematic representation of two (or several) magnetic devices (impurity atoms, adatoms, or magnetic molecules) dynamically coupled through a low-dimensional atomic lattice. Although a more accurate description ${ }^{24}$ is required to investigate the spin dynamics of such systems in detail, our computational model, which is transferable to first-principles methods, is an important step in this direction.

This paper is organized as follows. In Sec. II we introduce the model system and our theoretical framework. Section III contains the results of our investigations. First we investigate the electron response of an atomic wire, not including magnetic impurities, to local spin excitations. Second, we study the dynamical interplay between two spin impurities electronically connected by the wire and show how such a dynamics is affected by the gate voltage. Finally we draw some conclusions.

\section{THE MODEL}

A cartoon of the device considered is presented in Fig. 1. This consists of an $\mathrm{N}$-sites long atomic wire interconnecting two magnetic centers. The latter, represented in the figure by the spin vectors, enter our model as substitutional magnetic impurities positioned at the two ends of the wire. One of them, say the left-hand side spin center $\boldsymbol{S}_{1}$, is labeled as "driven," as its precession is induced and sustained by a local (at that site) magnetic field. The other localized spin, $\boldsymbol{S}_{2}$, located at the other end of the chain is the "probe" spin and it is not directly coupled to any external magnetic field but only to the electron gas. In this way $\boldsymbol{S}_{2}$ probes the magnetic excitations produced by the driven spin $S_{1}$ as these propagate through the interconnecting wire.

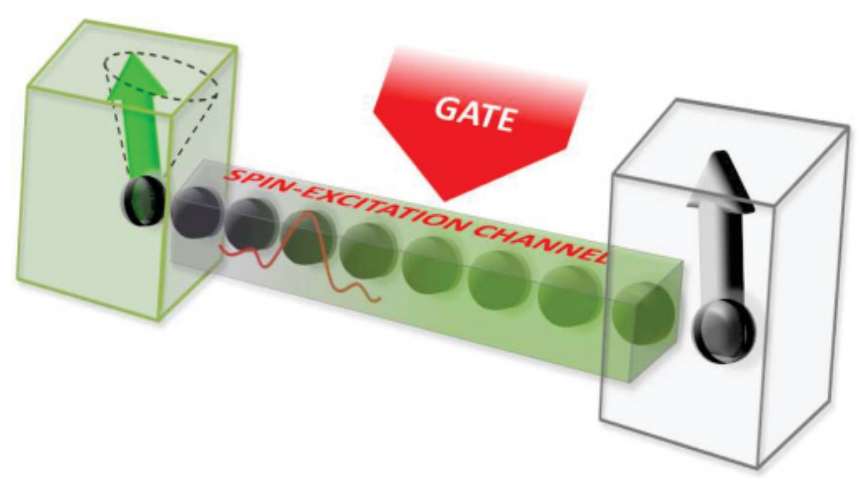

FIG. 1. (Color online) Model system investigated in this work: Two localized spins are electronically connected by a monoatomic wire, where electrons can flow. An electrostatic gate is applied to some of the interconnecting sites.

We describe the electronic structure of our device in the spirit of a two-spin-species model, e.g., the weakly interacting single-particle limit of the $s$ - $d$ model, ${ }^{25}$ where the magnetic moments of the impurities, originating from the deeply localized $d$ electrons, are exchange coupled to the conduction $s$ electrons. As outlined in the introduction, we follow the adiabatic (quasiclassical) spin dynamics approximation of Antropov, ${ }^{15}$ only at a more empirical level. The time-dependent Hamiltonians of the two exchange-coupled subsystems read

$$
\begin{aligned}
\hat{H}_{\mathrm{el}}(t)= & \sum_{\substack{i, j=1, N \\
\alpha=1,2}} H_{i j}^{\mathrm{TB}} c_{i}^{\alpha \dagger} c_{j}^{\alpha} \\
& -J \sum_{\substack{\alpha, \beta=1 \\
2}}\left[\boldsymbol{S}_{1}(t) c_{1}^{\alpha \dagger} c_{1}^{\beta}+\boldsymbol{S}_{2}(t) c_{N}^{\alpha \dagger} c_{N}^{\beta}\right] \cdot \hat{\boldsymbol{\sigma}}^{\alpha \beta}, \\
H_{\mathrm{S}}(t)= & -\boldsymbol{S}_{1}(t) \cdot\left[J \boldsymbol{S}_{1}(t)+g \mu_{\mathrm{B}} \boldsymbol{B}\right]-J \boldsymbol{S}_{2}(t) \cdot \boldsymbol{s}_{N}(t) .
\end{aligned}
$$

The top expression is for the quantum electrons. Here $H_{i j}^{\mathrm{TB}}=$ $\varepsilon_{i} \delta_{i j}+\gamma \delta_{i, i \pm 1}$ is a single-orbital TB Hamiltonian with onsite energies $\varepsilon_{i}$ and hopping integral $\gamma(\gamma$ sets the relevant energy scale for the entire system); $c_{i}^{\alpha \dagger}\left(c_{i}^{\alpha}\right)$ is the creation (annihilation) operator for an electron with spin-up $(\alpha=1)$ or spin-down $(\alpha=2)$ at the atomic site $i ; \hat{\boldsymbol{\sigma}}=\frac{1}{2}\left(\sigma_{x}, \sigma_{y}, \sigma_{z}\right)$ is the electron spin operator, $\left\{\sigma_{l}\right\}_{l=x, y, z}$ being the set of Pauli matrices; $J>0$ is the exchange coupling strength.

The classical Hamiltonian $H_{S}(t)$ describes the interaction of the local spins with the mean-field local electron spin density $\boldsymbol{s}_{i} \equiv\langle\hat{\boldsymbol{\sigma}}\rangle_{i}$ taken as the instantaneous expectation value of the conduction-electron spin at site $i$ (see further in text for the exact definition). The magnetic-field-like object $\boldsymbol{B}=\left(0,0, B_{z}\right)$ in the first term does not imply any true physical meaning of an extremely localized external magnetic field. This is only an instrumental variable that we use to drive the precession of $\boldsymbol{S}_{1}$ which gives rise to spin pumping in the system. We assume $g=2$ for the localized spins and $\mu_{B}=5.788 \times 10^{-5} \mathrm{eV} / \mathrm{T}$ is the Bohr magneton.

We then investigate in the time domain what is essentially the spin analog of the Ehrenfest molecular dynamics ${ }^{19}$ by integrating the set of coupled quantum and classical equations 
of motion (EOM) of the two spin subsystems, ${ }^{26-28}$ as they result from the Hamiltonian dynamics

$$
\begin{aligned}
\frac{d \hat{\rho}}{d t} & =\frac{i}{\hbar}\left[\hat{\rho}, \hat{H}_{\mathrm{el}}\right], \\
\frac{d \boldsymbol{S}_{n}}{d t} & =\left\{\boldsymbol{S}_{n}, H_{\mathrm{S}}\right\}=\frac{\boldsymbol{S}_{n}}{S} \times \begin{cases}J \boldsymbol{s}_{1}+g \mu_{\mathrm{B}} \boldsymbol{B} & \text { for } n=1 \\
J \boldsymbol{s}_{N} & \text { for } n=2\end{cases}
\end{aligned}
$$

Here $\{$,$\} represents the classical Poisson bracket, [,] stands$ for the quantum-mechanical commutator, and $S=\hbar$ is the magnitude of the two classical spins. The first EOM is for the electron density matrix $\hat{\rho}$. At the initial time, $t_{0}, \hat{\rho}$ is constructed from the eigenstates $\left\{\left|\varphi_{\nu}\right\rangle\right\}_{\nu=1}^{2 N}$ of the spinpolarized electron Hamiltonian $\hat{H}_{\mathrm{el}}\left(t_{0}\right)$ [see Eq. (1)], with the composite index $v=\{i, \sigma\}$ labeling the set of $2 N$ spinpolarized eigenstates. We define $\rho\left(t_{0}\right)=\sum_{v} f_{\nu}\left|\varphi_{\nu}\right\rangle\left\langle\varphi_{\nu}\right|$ with $f_{v}=\eta_{F}\left(\epsilon_{v}-E_{F}\right)$ being the occupation numbers distributed according to Fermi-Dirac statistics. The instantaneous on-site spin density is thus generated as

$$
\boldsymbol{s}_{i}(t) \equiv\langle\boldsymbol{\sigma}\rangle_{i}(t)=\operatorname{Tr}[\rho(t) \boldsymbol{\sigma}]_{i}=\sum_{\alpha \beta} \rho_{i i}^{\alpha \beta}(t) \boldsymbol{\sigma}^{\beta \alpha} .
$$

The coupled EOMs are integrated numerically by using the fourth-order Runge-Kutta (RK4) algorithm. ${ }^{29}$ As a result the set of trajectories for the local electronic spin densities $s_{i}(t)$ are obtained as well as those of the driven and the probe classical spins $S_{1,2}(t)$. The typical length of the chain that we consider is $N=100$.

The effect of an electrostatic gate is incorporated in our model as a rigid shift of the on-site energies $\varepsilon_{i} \rightarrow \varepsilon_{i}+V_{g}$, where $i \in\left[i_{1}, i_{2}\right]$ is a certain range of sites in the middle of the chain and $V_{g}$ is the gate voltage. ${ }^{34}$

\section{DYNAMICS OF THE ITINERANT SPINS WITH FROZEN IMPURITIES}

\section{A. No external gate}

As a preliminary step toward the combined quantumclassical dynamics we first address the dynamics of the spin density of the itinerant electrons in the presence of the two local spins, whose directions are fixed, e.g., $\boldsymbol{S}_{1,2} \| \hat{\boldsymbol{z}}$. A spin excitation is produced by a small but finite spatially localized perturbation in the spin density. As initial density matrix at $t_{0}$ we use the one that corresponds to a perturbed Hamiltonian $\hat{H}_{\mathrm{el}}\left(t_{0}-\delta t\right)$ in which one of the localized spins is slightly tilted in the $x$ - $z$ plane, such that $S_{1}\left(t_{0}-\delta t\right) \times \hat{z} /\left|S_{1}\right| \approx d \theta \lesssim 5^{\circ}$. However at $t_{0}$ we bring $S_{1}$ back to its original direction $(\| \hat{z})$ where it stays throughout the simulation. In other words we study the time evolution of the system with the two local spins parallel to each other starting from the ground-state electronic charge density of the system where one of the two spins is tilted by a small angle.

The evolution of the density matrix for $t>t_{0}$ is then given by $\hat{\rho}(t)=e^{-i \hat{H}_{\mathrm{el}} t / \hbar} \hat{\rho}\left(t_{0}\right) e^{i \hat{H}_{\mathrm{el}} t / \hbar}$, which translates into the following expression for the matrix elements of the density matrix written over the basis of the eigenvectors $\left|\varphi_{n}\right\rangle$ of the Hamiltonian $\hat{H}_{\mathrm{el}}\left(t_{0}\right)$ :

$$
\rho_{m n}(t)=e^{-i \omega_{m n} t}\left\langle\varphi_{m}\left|\hat{\rho}\left(t_{0}\right)\right| \varphi_{n}\right\rangle \equiv e^{-i \omega_{m n} t} \rho_{m n}\left(t_{0}\right) .
$$

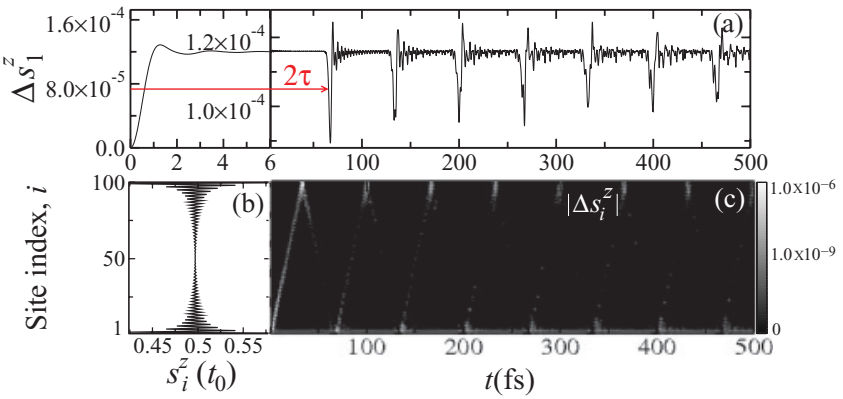

FIG. 2. (Color online) Spin excitation of one-dimensional wire. (a) Time dependence of the spin polarization on the first site $\left(\Delta s_{1}^{z}\right)$ as obtained from the numerical integration of the EOMs and directly from Eq. (6). (b) Initial spin polarization $s^{z}\left(t_{0}\right)$ as a function of site index. (c) Time and space evolution of $\Delta s_{i}^{z}$ with the color shade representing the magnitude of absolute value of $\Delta s_{i}^{z}(t)$. Note that $2 \tau$ is the wave-packet round-trip time as also seen in the bottom panel.

In Eq. (5) we have defined the frequencies $\omega_{m n} \equiv\left(e_{m}-e_{n}\right) / \hbar$ corresponding to differences between the eigenvalues $e_{n}$ of $\hat{H}_{\mathrm{el}}\left(t_{0}\right)$. Note that the initial density matrix at $t=t_{0}$ corresponds to the perturbed Hamiltonian $\hat{H}_{\mathrm{el}}\left(t_{0}-\delta t\right)$. In order to investigate the transport of spins along the chain we use the above expression projected onto the basis of the spin-polarized local TB atomic orbitals $|i \sigma\rangle$, where $i$ represents the atomic site and $\sigma=\uparrow, \downarrow$ is the spin component:

$$
\rho_{k l}^{\sigma \sigma \prime}(t)=\sum_{m n} e^{-i \omega_{m n} t} c_{k m}^{\sigma} c_{l n}^{\sigma *} \sum_{i \alpha} \sum_{j \beta} c_{i m}^{\alpha *} c_{j n}^{\beta} \rho_{i j}^{\alpha \beta}\left(t_{0}\right) .
$$

In Eq. (6) the coefficients $c_{i n}^{\sigma} \equiv\left\langle i \sigma \mid \varphi_{n}\right\rangle$ are the projections of the eigenvectors $\left|\varphi_{n}\right\rangle$ on the spin-resolved atomic orbitals $|i \sigma\rangle$.

The typical evolution of the spin density of the itinerant electrons resulting from the excitation described above is presented in Fig. 2. The trajectories of the individual onsite spin polarizations $\Delta s_{i}^{z}(t)=s_{i}^{z}(t)-s_{i}^{z}\left(t_{0}\right)$ stemming from Eq. (6) are perfectly identical (on the scale of the graph) to those obtained by the numerical integration of the EOM [i.e. from Eq. (3)], confirming the reliability of our time integrator for the typical duration of the simulations.

The spatial distribution of the initial spin polarization, $s_{i}^{z}\left(t_{0}\right)$, is shown in Fig. 2(b) and its subsequent evolution, presented in Fig. 2(c) can be qualitatively characterized as the propagation of a spatially localized spin wave packet. This travels along the wire with a practically uniform velocity very close to the Fermi level group velocity, as expected in view of the rather small overall local spin polarization of the chain. The packet then gradually loses its sharpness as it disperses. However, the backbone of the packet is detectable even after a few tens of reflections at the ends of the wire. Such a feature demonstrates that this finite 1D model atomic system is a relatively efficient waveguide for spin wave packets (of sub-femtosecond duration) at least over the investigated time scales of a few picoseconds.

Equation (6) can also be used to calculate directly the spectra of particular spin observables. These match perfectly with those calculated by performing the discrete Fourier transform $^{30}$ (dFT) of the time-dependent spin densities obtained over the finite duration of the dynamic simulation. 

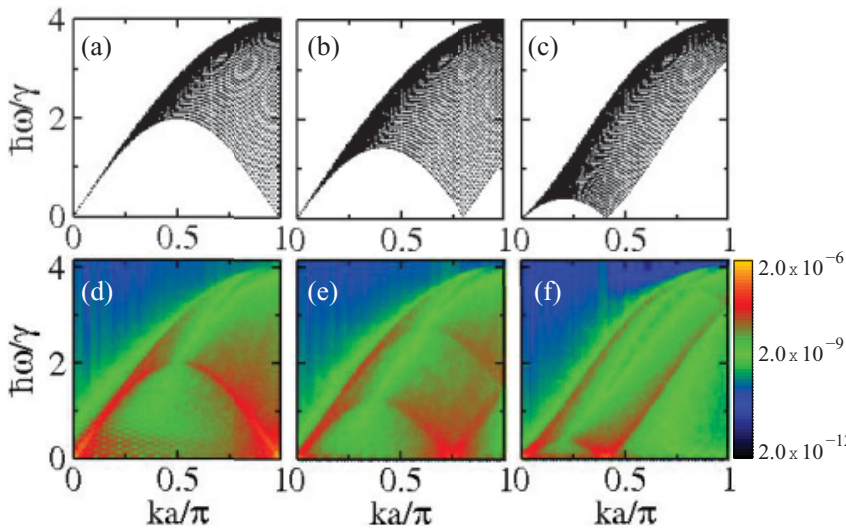

FIG. 3. (Color online) $\operatorname{dFT}\left[\Delta s_{i}^{z}(t)\right](k, \omega)$ for different band filling: (a), (d) $\rho_{0}=0.5$; (b), (e) $\rho_{0}=0.6$; and (c), (f) $\rho_{0}=0.8$. The top panels show the exact analytical excitation spectrum for a homogeneous TB chain without local spins. The bottom panels show the results of our numerical simulations. The intersite distance $a$ is arbitrary. The color shade in the bottom panel represents the absolute value of $\operatorname{dFT}\left[\Delta s_{i}^{z}(t)\right](k, \omega)$ and is logarithmically scaled for better contrast.

Clearly, in the absence of the localized impurities (i.e., for a finite homogeneous TB chain) $\omega_{m n}$ can be calculated exactly. The spectrum of $\Delta s_{i}^{z}$ has monotonously decreasing amplitudes from the lowest possible frequency $\omega_{\min } \approx \frac{3 \pi^{2}|\gamma|}{(N+1)^{2} \hbar}$ to the maximum one $\omega_{\max } \approx \frac{4|\gamma|}{\hbar}$ (expressed in the limit of $N \rightarrow \infty)$. This is also true for the case of interaction with the two frozen local impurities (e.g., for $J=\gamma$ ) as these have a minor effect on the electron Hamiltonian. For the parameters typically used in our simulations the corresponding maximum period $T_{\max }=\frac{2 \pi}{\omega_{\min }} \lesssim 1$ ps is within the total time of the simulation while the corresponding minimum period $T_{\min }=\frac{2 \pi}{\omega_{\max }} \approx 1 \mathrm{fs}$ is much larger than the typical time step $\Delta t \stackrel{\omega_{\max }}{=} 0.01 \mathrm{fs}$.

A more detailed spectral analysis is obtained by the twodimensional dFT power portraits ${ }^{30}$ of $\Delta s_{i}^{z}$ (see Fig. 3) denoted as $\mathrm{dFT}\left[\Delta s_{i}^{z}(t)\right](k, \omega)$. In Fig. 3 we compare such spectra for the itinerant spin dynamics to its exact counterpart in the case of a finite homogeneous TB chain. These portraits reveal key features of the one-dimensional fermionic system ${ }^{31}$ that vary systematically with the band filling $\rho_{0}$, namely (i) a near continuum of allowed modes in a certain $(k, \omega)$-space region, defined by a low- and a high-energy dispersion functions, and (ii) a linear dispersion for small $k(\omega \propto k$ for $k \rightarrow 0$ ). Analogous mode-occupation patterns have been rigorously analyzed in relation to the dynamical properties of one-dimensional quantum Heisenberg spin chains which too have a TB type dispersion relation. ${ }^{32}$ The low-energy mode-occupation limit is due to the fact that the energy of the electron-hole excitation can approach zero only for $\Delta k \rightarrow 0$ and $\Delta k \rightarrow 2 k_{\mathrm{F}}$, where $k_{\mathrm{F}}$ is the Fermi vector $\left(k_{\mathrm{F}}=\frac{\pi}{2 a}\right.$ for $\rho_{0}=0.5$, corresponding to one electron per site). The variation of the band filling away from the half filling results in a folding of the low-energy limit as shown in Figs. 3(b), 3(c), 3(e), and $3(f)$. Note that due to electron-hole symmetry we only show the spectra for $\rho_{0} \geqslant 0.5$.

\section{B. Electrostatic gate applied}

The dynamics of the itinerant spins, as described by Eq. (5), in the case of an electrostatic gate applied to a section of the wire (typically in the middle of the wire) cannot be expressed in a closed form as a function of $V_{g}$ for arbitrarily big systems. As such we resort to our numerical integration scheme. Before addressing the dynamics, however, we first analyze the groundstate electronic structure of the gated wires for different values of the gate potential $V_{g}$. Displayed in Fig. 4 is the adiabatic variation of the eigenvalues for a non-spin-polarized chain with $N=30$ sites (without localized spins) as a function of the gate potential $V_{g}$ (the gate is applied to 10 sites in the middle of the chain, i.e., at the sites with indexes going from $i_{1}=11$ to $i_{2}=20$ ). Note that energy-related quantities on all the figures are in units of the hopping integral $\gamma$. For $V_{g}$ close to 0 , the discrete energy spectrum spans in the range $[-2 \gamma, 2 \gamma]$. With the increase of $V_{g}$ the level spacing distorts as the eigenstates are affected differently by the gate. Certain eigenvalues grow nearly linearly with increasing $V_{g}$ (these are shown as red squares in Fig. 4). The spatial distribution of these eigenstates is predominantly concentrated in the gated region; i.e., they correspond to the region where the local on-site energy has been modified.

For extremely large values of $V_{g}\left(V_{g}>4 \gamma\right)$ the chain is effectively split into three energetically decoupled parts, the gated middle and the two identical unbiased ends on the left-hand side and on the right-hand side. More interesting for us is the range of intermediate gate voltages, for which the three parts of the wire are substantially affected but not yet decoupled by the gate voltage. For such $V_{g}$ avoided crossings occur between states localized in the gated and nongated

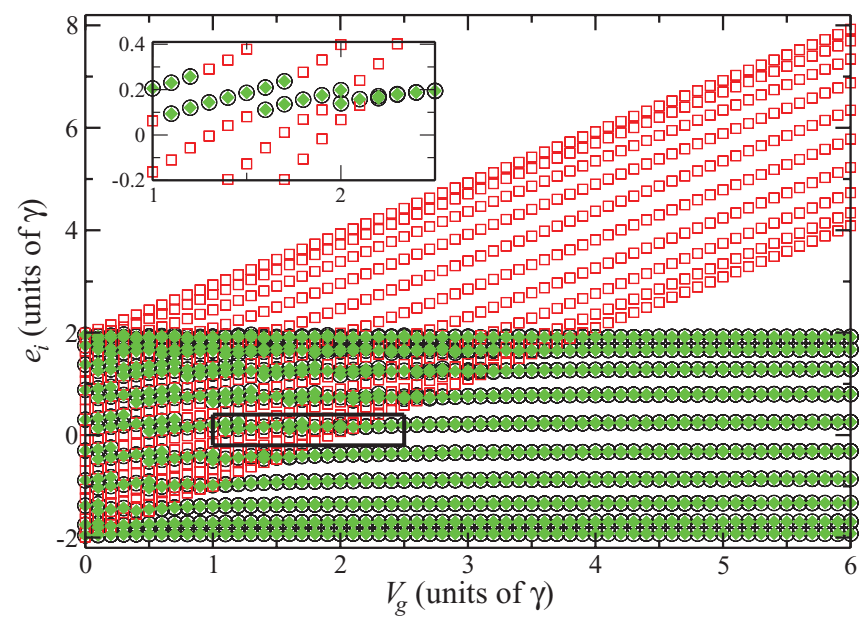

FIG. 4. (Color online) Schematic of the gate dependence of the ground-state energy spectrum of $\hat{H}_{\mathrm{el}}$. According to their weights at the three subsections of the chain $\Omega_{\mathcal{L}, \mathcal{M}, \mathcal{R}}=\sum_{i \in \mathcal{L}, \mathcal{M}, \mathcal{R}}\left|c_{\text {in }}\right|^{2}$ we distinguish three types of states depending on which of the three partial weights is the greatest. We use black circles for the case of $\Omega_{\mathcal{L}}$, red squares for $\Omega_{\mathcal{M}}$, and green diamonds for $\Omega_{\mathcal{R}}$. Note that the eigenstates corresponding to the two ungated regions $\left(\Omega_{\mathcal{L}}\right.$ and $\left.\Omega_{\mathcal{R}}\right)$ are degenerate by symmetry, as the gate is applied in the exact center of the wire. The inset shows a magnification of an area of intermediate $V_{g}$, illustrating the situation of avoided crossings. A short chain with $N=30$ sites is used for simplicity. 


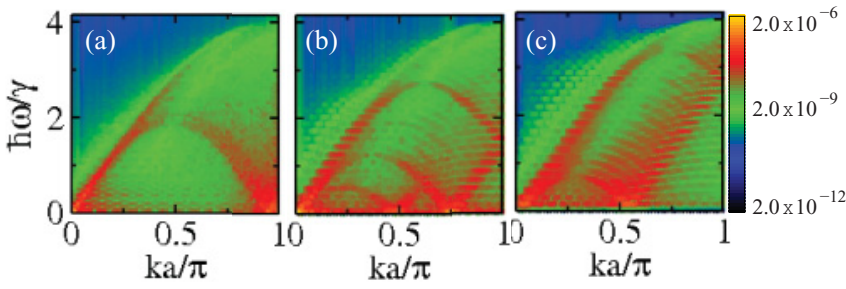

FIG. 5. (Color online) $\mathrm{dFT}\left[\Delta s_{i}^{z}(t)\right](k, \omega)$ for different values of the gate potential: (a) $V_{g}=0.2 \gamma$, (b) $V_{g}=2.2 \gamma$, and (c) $V_{g}=4.6 \gamma$. The color shade is identical to that in Fig. 3.

regions. This gives rise to additional low-frequency lines in the dynamical spectrum as described by Eq. (6). As we will demonstrate in the Appendix the presence of these avoided crossings around the Fermi level for certain intermediate $V_{g}$ yields an enhanced transmission through the gated wire (waveguide) at low frequencies.

The two-dimensional dFT images (Fig. 5) of the spin dynamics in the presence of the gate bring additional dimension to the electron spectroscopy analysis. The difference here with respect to the case depicted in Fig. 3(d) is that a gate has been applied to the middle of the chain in the ground state; i.e., $V_{g} \sum_{\alpha, i \in\left[i_{1}, i_{2}\right]} c_{i}^{\alpha \dagger} c_{i}$ has been added to $\hat{H}_{\mathrm{el}}$ at $t=t_{0}$. Again we use the half-filling case, $\rho_{0}=0.5$ (one electron per atom). From the figure it is immediately noticeable that the effect of the variation of $V_{g}$ on the excitation spectra is quite similar to the effect of the band filling in the nongated case. Due to the gate potential, the relative electron populations of the gated and gate-free parts of the chain change (the gated region is depopulated). For intermediate values of $V_{g}$ [see Fig. 5(b)] the excitation spectrum is rather a superposition of two spectra with band fillings below and above 0.5 (approximately 0.2 and $0.6)$. Furthermore we find a substantially increased population of the low-frequency modes for all $k$ vectors, i.e., of states forbidden by symmetry for $V_{g}=0$. For large $V_{g}$ [see Fig. 5(c)] the middle part of the wire becomes almost completely depleted and the $(k, \omega)$ portrait corresponds effectively to the excitation spectrum of a single chain with a band filling $\rho_{0} \approx 0.75$, which is similar to the nongated case presented in Fig. 3(f).

\section{COMBINED QUANTUM-CLASSICAL SPIN DYNAMICS IN THE PRESENCE OF A GATE}

The inclusion of dynamic local spin impurities in the model requires the numerical integration of the set of coupled nonlinear EOMs of Eq. (3). For a small number of classical spins the dynamics of the itinerant spin density is qualitatively very similar to what is described by Eq. (6). The frequencydomain analysis of the classical spin trajectories by means of dFT reveals the characteristic signature of the discrete electronic spectrum with only additional modulations in the amplitudes. We focus now on the case in which $S_{1}$ is driven by a local magnetic field into a precession, hence it acts as a spin-pump (see cartoon in Fig. 1). It is important to note that what we refer to as a local magnetic field $\boldsymbol{B}=\left(0,0, B^{z}\right)$ is only instrumental to trigger and sustain a uniform Larmor precession of $\boldsymbol{S}_{1}$; i.e., it does not produce any Zeeman splitting in the itinerant electrons spectrum. We consider now the
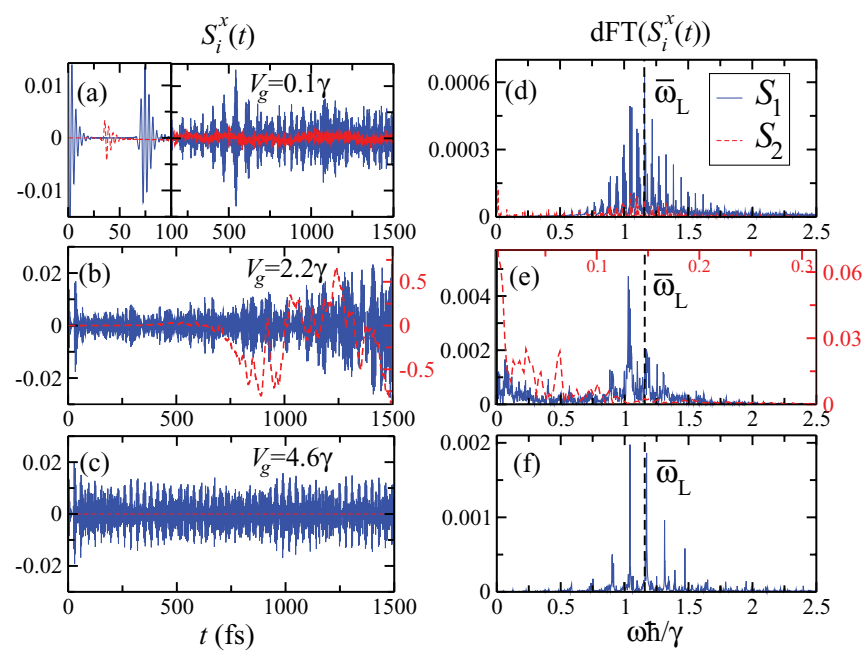

FIG. 6. (Color online) Time evolution of the $x$ components of the localized spins, $S_{1}^{x}(t)$ (blue solid line) and $S_{2}^{x}(t)$ (red dashed line) [left] and the corresponding spectra, $\operatorname{dFT}\left[S_{i}^{x}(t)\right](\omega)$, [right] for three different gate voltages (a), (d) $V_{g}=0.1 \gamma$; (b), (e) $V_{g}=2.2 \gamma$; and (c), (f) $V_{g}=4.6 \gamma$. The frequency domain is represented by the dimensionless quantity $\hbar \omega / \gamma$ and $\bar{\omega}_{L}=\hbar \omega_{L} / \gamma=1.16$ for $\gamma=1 \mathrm{eV}$. Note that in the case of $V_{g}=2.2 \gamma$ different scales are used for the magnitudes of $S_{1}^{x}(t)$ and $S_{2}^{x}(t)$ and their spectra [axes corresponding to $S_{2}^{x}(t)$ are marked in red and are shown on the opposite sides of the graph].

following situation: The quantum-classical spin system is in its ground state until $t=t_{0}$, when the first local spin $\boldsymbol{S}_{1}$ starts fluctuating to form a small misalignment with $\boldsymbol{B}$. This sets the entire quantum-classical spin system into motion. The typical trajectories of the transverse components $S_{1}^{x}(t)$ and $S_{2}^{x}(t)$ and their frequency-domain dFT images for different values of the gate voltage are presented in Fig. 6.

In this case the excitation of a transverse spin density at $t=0$ by the initiation of the Larmor precession is very similar in nature to the excitation induced by tilting one local spin investigated before (Sec. III). In fact it indeed develops in a very similar way in the early stages of the time evolution. Just like in Fig. 2 a nonequilibrium transverse spin-density spin packet is sent along the wire and both the localized spins respond each time the packet reaches them. Figure 6(a) displays the case of a very small gate voltage $V_{g} \ll \gamma$. The frequency-domain image of the precessional motion of $S_{1}^{x}(t)$ has the signature of the discrete electronic spectrum with an amplitude envelope that peaks at the Larmor frequency $\omega_{\mathrm{L}}=$ $2 \mu_{B} B_{1} / \hbar$. The dFT-portrait of the probe spin $S_{2}^{x}(t)$ is very similar to that of $S_{1}^{x}(t)$. Although significantly down-scaled in amplitude, it has the electronic-structure modes imprinted in its classical motion in the same way as it is for the driven spin. This qualitative behavior is observed for $V_{g}=0$ and a range of small voltages $V_{g} \leqslant \gamma$ but it changes dramatically for gate voltages $V_{g}>\gamma$ [see Figs. 6(b) and 6(c)]. The case of extremely big voltages $V_{g}>4 \gamma$ (depicted in the bottom panel) is a trivial one for which the electrostatic barrier $V_{g}$ is completely opaque to the electrons and the probe spin $\boldsymbol{S}_{2}$ does not respond to the Larmor precession of $\boldsymbol{S}_{1}$ (note also the rarefaction of modes in the $S_{1}^{x}$ spectrum due to the effective shortening of the chain by $2 / 3$ ). 

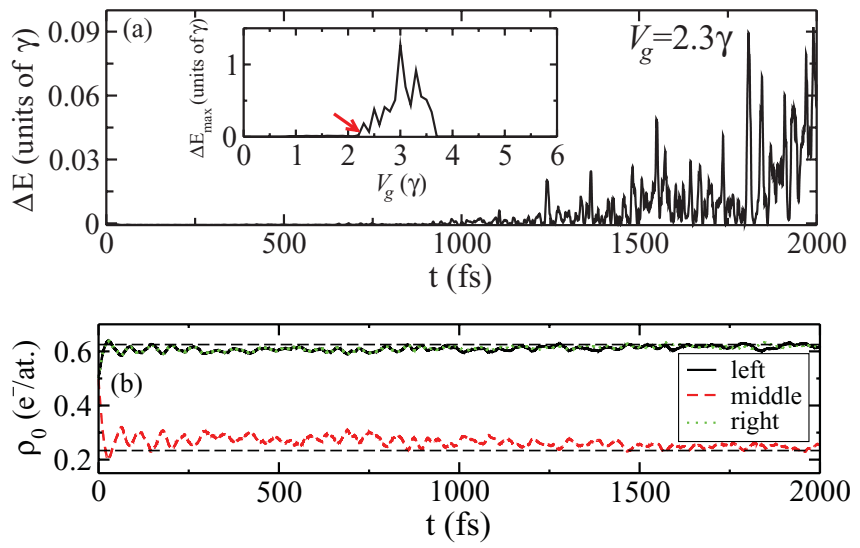

FIG. 7. (Color online) (a) Time evolution of the energy transfer, $\Delta E(t)$, between electronic and localized spins for $V_{g}=2.2 \gamma$. The inset shows the maximum value of the energy transfer, $\Delta E_{\max }$, for a fixed duration of simulation $(2 \mathrm{ps})$ as a function of $V_{g}$. A red arrow indicates the particular voltage for which $\Delta E(t)$ is plotted. (b) Evolution of the electron populations of different parts of the chain for $V_{g}=2.2 \gamma$ : left (black solid line), middle (red dashed line), and right (green dotted line). The two black dashed horizontal lines show the equilibrium populations of the gate-free parts (upper line) and of the gated middle section (lower line), i.e., the populations corresponding to the case when the gate is applied in the ground state.

Special attention must be devoted to the intermediate $V_{g}$ regime in which the dynamics of the probe spin is qualitatively different and uncorrelated to that of the driven one [see Fig. 6(b)]. The typical outcome of the real-time dynamics at such intermediate voltages $\gamma<V_{g}<4 \gamma$ is that the probe spin starts accumulating very big transverse deflections. This is then fed back into the pumping spin which also deflects more but still preserves, to a great extent, its precession about the magnetic field.

It is worth noting that this self-amplification of the spin dynamics does not come at a total energy cost, as the model system described by Eq. (3) is completely conservative. As such we simply observe a conversion of electrostatic energy into "spin energy" in the form of a spin amplitude transverse to the driving magnetic field. This accumulation is related to the increasing energy transfer from the itinerant electrons to the localized moments, as illustrated in Fig. 7(a). Such an energy transfer is defined as $\Delta E(t)=$ $\left|\Delta E_{\mathrm{el}}(t)-\Delta E_{\mathrm{S}}(t)\right|$, where $\Delta E_{\mathrm{el} / \mathrm{S}}(t)=E_{\mathrm{el} / \mathrm{S}}(t)-E_{\mathrm{el} / \mathrm{S}}\left(t_{0}\right)$, $E_{\mathrm{el}}(t)=\operatorname{Tr}\left[\rho(t) H_{\mathrm{el}}(t)\right]$, and $E_{\mathrm{S}}(t)=-g \mu_{B} \boldsymbol{S}_{1}(t) \cdot \boldsymbol{B}$. The total energy conservation $\Delta E_{e l}(t)=-\Delta E_{S}(t)$ has been verified within a relative error of $10^{-9} \%$. The amplification of the energy transfer ${ }^{35}$ is characteristic of this $V_{g}$ range $\left(\gamma<V_{g}<\right.$ $4 \gamma$ ) [see the inset of Fig. 7(a)]. The frequency-domain image of the localized spin trajectories [see Fig. 6(e)] for $\gamma<V_{g}<4 \gamma$ shows a substantial qualitative difference for the driven and the probe spins. While around $\omega_{\mathrm{L}}$ the shape of the spectrum of $\boldsymbol{S}_{1}$ is similar to the two extreme $V_{g}$ cases, a significant amplitude is accumulated now at low frequencies. The main difference from the small $V_{g}$ case, however, is in the spectrum of $\boldsymbol{S}_{2}$ which shows practically no response at the Larmor frequency but has very large amplitudes in the low frequency range.

This behavior in the classical spin dynamics, characteristic of certain gate voltages, stems from the dominance of the
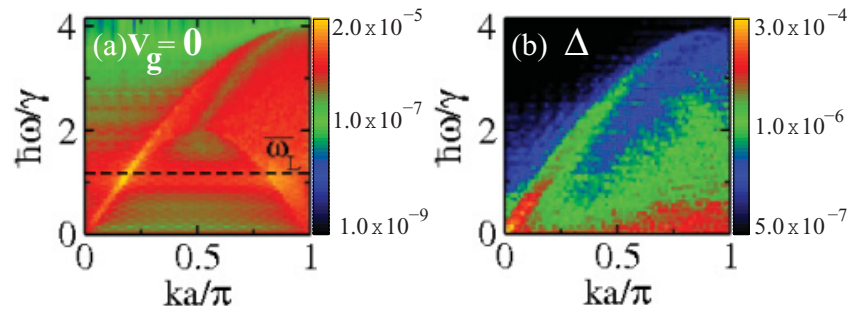

FIG. 8. (Color online) (a) $\operatorname{dFT}\left[s_{i}^{x}(t)\right](k, \omega)$ for $V_{g}=0$. (b) The difference between $\operatorname{dFT}\left[s_{i}^{x}(t)\right](k, \omega)$ for $V_{g}=2.2 \gamma$ and for $V_{g}=0$ defined as $\Delta=\left|\operatorname{dFT}\left[s_{i}^{x}(t)\right](k, \omega)_{V_{g}=2.2 \gamma}-\operatorname{dFT}\left[s_{i}^{x}(t)\right](k, \omega)_{V_{g}=0}\right|$. The color shade represents the absolute value of $\operatorname{dFT}\left[s_{i}^{x}(t)\right](k, \omega)$ and $\Delta$, respectively, and is logarithmically scaled for better contrast.

low-frequency modes in the electron dynamics at those $V_{g}$. The effect can be seen also in the much simpler case of a non-spin-polarized uniform atomic wire subjected to a sudden switch of an electrostatic gate in the middle. In the Appendix we consider the charge dynamics associated to this situation and analyze the lowest frequency components entering in Eq. (6). The initial charge excitation, similarly to the transverse spin polarization in the model above, is triggered by a local electrostatic perturbation at the first site. It follows from Eq. (6) that, for certain values of $V_{g}$ for which two adjacent adiabatic eigenenergies $\left(e_{i}, e_{i+1}\right)$ around the Fermi level come closer to each other in an avoided crossing, the dynamical charge amplitude at the opposite end of the wire exhibits a peak at that particular very low frequency $\left(e_{i+1}-e_{i}\right) / \hbar$. In other words, a certain gate voltage condition is created for resonant charge transfer between the three parts of the chain at low frequencies. This, in the case of itinerant transverse spin polarization, promotes the enhanced reaction of the probe spin to the spin pumping produced on the other side of the gate.

The electronic part of full quantum-classical spin dynamics in the presence of the gate (Fig. 6) is, in many respects, similar to the charge-only case, described in Sec. III B and in the Appendix. As before, we construct the two-dimensional dFT portraits of the spin-density evolution. Providing additional $k$-resolved information, these give another perspective for interpreting the dramatic increase of the lowfrequency oscillations of the probe spin at certain gate voltages, when compared to the case of frozen $\boldsymbol{S}_{i}$ and electrons at $t=t_{0}-\delta t$ relaxed to the external gate potential (Fig. 5). In the latter case, the increased low-frequency occupations (with respect to the nongated case) were attributed to the split of the one-dimensional system into subsystems with different average electron densities. Here we find a similar dFT pattern, now in the presence of dynamically coupled local spins and a gate abruptly introduced at $t=t_{0}$. Those two additional time-dependent factors result in a significant variation in time of the electron distributions in the gated and nongated segments of the chain [see Fig. 7(b)]. The dFT image in this case can be interpreted as a superposition of spectra corresponding to different $\rho_{0}$, resulting in a smearing of the low-frequency structure previously present in the $(k, \omega)$-space image. A comparison of the results for $V_{g}=0$ and $V_{g}=2.2 \gamma$ is presented in Fig. 8. We find that the full dynamical $V_{g}=0$ portrait is rather similar to the results obtained for frozen impurities (see Fig. 5), the only additional feature being the 
adiabatic excitation at the Larmor frequency of the driven spin. The difference (point-by-point subtraction of the contour plots) with the corresponding gated case, depicted in Fig. 8(b), shows that it is indeed mainly the lower frequency band of the spectrum (for any wave vector) that gains occupation in the gated case.

\section{CONCLUSIONS}

In summary, we have employed atomistic dynamical simulations to investigate the effect of an electrostatic gate as a means of controlling the indirect coupling between two distant localized spin impurities in a finite metallic wire comprising one hundred atoms. One of the impurities, precessing in external magnetic field, plays the role of a spin pump and the response of the second (probe) spin was analyzed as a function of the gate potential applied to the interconnecting wire. We identified a range of external gate potentials for which the spin pumping is extremely efficient and leads to a substantial excitation of transversely polarized itinerant spin density in the nongated parts of the chain. This, in turn, produces a massive low-frequency swing of the probe spin. Such a resonant effect has been related to gate-induced avoided crossings in the electronic structure of the interconnect. For certain gate voltages these occur in the vicinity of the Fermi level and assist the enhanced transfer of charge or spin across the barrier. Evidence for this effect is also identified in the Fourier portraits of the calculated time-dependent spin distribution.

\section{ACKNOWLEDGMENTS}

This work is sponsored by Science Foundation of Ireland (Grant No. 07/IN.1/I945). Computational resources have been provided by the Trinity Center for High Performance Computing and by the Irish Center for High-End Computing.

\section{APPENDIX: THE CHARGE-ONLY ANALOG-QUASIANALYTIC SOLUTION}

We consider the eigenstate-resolved dynamical amplitude $A_{m n}$ corresponding to a mode with frequency $\omega_{m n}$, associated with the transitions between the eigenstates $e_{m}$ and $e_{n}$. The system is the simplified wire used for the spectroscopic analysis of Fig. 4; i.e., it is a non-spin-polarized 30-atom-long chain. Analogously to the spin-polarized case, the $t=t_{0}$ excitation is applied simply as a potential shift at the first atomic site. From Eq. (6) the dynamically driven chargeaccumulation amplitude at site $i$ is defined as

$$
A_{m n}^{i}=c_{i m} c_{\mathrm{in}}^{*} \sum_{k l} c_{k m}^{*} c_{l n} \rho_{k l}\left(t_{0}\right)
$$

such that

$$
\rho_{i i}(t)=\sum_{m n} A_{m n}^{i} e^{-i \omega_{m n} t}
$$

is the dynamic electron occupation at site $i$. This quantity is calculated at the last site, $i=N$, i.e., at the opposite side of the chain with respect to the perturbation. In Eq. (A1) $c_{i m}=\left\langle i \mid \varphi_{m}\right\rangle$ are the on-site components of the eigenvectors $\left|\varphi_{m}\right\rangle$ of the non-spin-polarized Hamiltonian $\hat{H}_{\mathrm{el}}\left(V_{g}\right)$ for which the gate voltage $V_{g}$ is applied (between the sites $i=11$ and $i=21$ ).

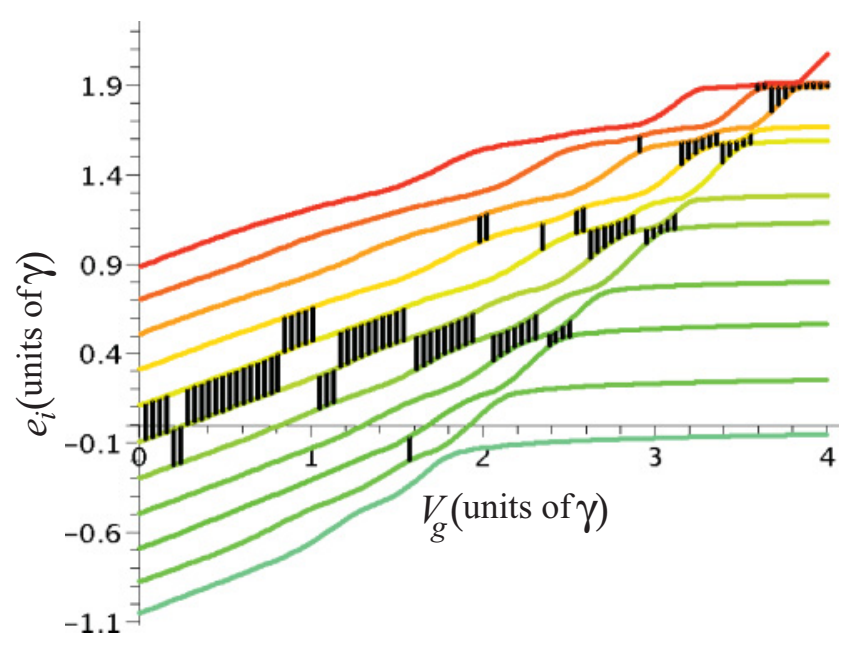

FIG. 9. (Color online) Adiabatic variation with the gate voltage of a few eigenvalues around the Fermi level (we have used $\varepsilon_{0}=0$ and $|\gamma|=1 \mathrm{eV}$, hence $E_{\mathrm{F}}=0$ at $V_{g}=0$ ). Marked with black lines are the pairs of eigenstates $(i, i+1)$ which give rise to the highest (in absolute value) dynamical amplitude $A_{i, i+1}^{N}$ at the last site $N$.

From all the pairs of adjacent eigenstates $\left(e_{i}, e_{i+1}\right)$, which give rise to the lowest frequency modes $\left[\omega_{i, i+1}=\left(e_{i+1}-\right.\right.$ $\left.\left.e_{i}\right) / \hbar\right]$ in the dynamics, we identify in Fig. 9 those which have the highest (in absolute value) amplitude $A_{i, i+1}^{N}$. For small gate voltages these are the eigenstates around the Fermi level but as $V_{g}$ increases the modes with the largest amplitudes tend to arise where avoided crossings of eigenstates occur. These are also the modes with frequencies close to the lowest possible frequency for that particular gate voltage.

The actual highest amplitude at the last site, corresponding to the highlighted modes in Fig. 9, are depicted as a function of $V_{g}$ in Fig. 10(a), together with the quantity $A_{\text {sum }}^{N} \equiv \sqrt{\sum_{i}\left(A_{i, i+1}^{N}\right)^{2}}$. This represents the pessimistic estimate (considering all modes orthogonal in phase) of the

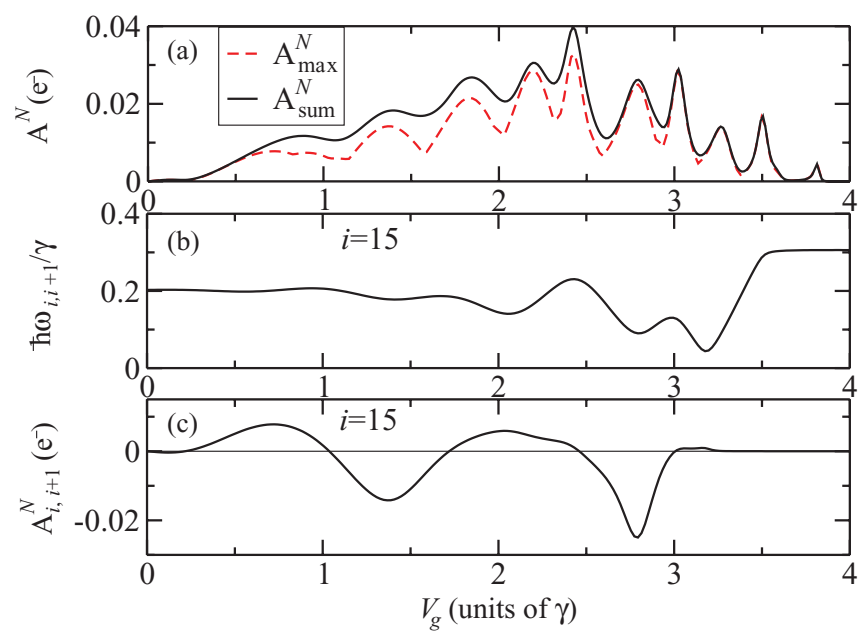

FIG. 10. (Color online) (a) The maximum single-mode amplitude $A_{\max }^{N}$ and an estimate for the total low-frequency amplitude $A_{\text {sum }}^{N}$ (see text for details) as a function of the applied gate voltage. (b) The frequency of the mode related to the pair of eigenstates $(i=15)$ around the Fermi level, adiabatically evolved with the gate voltage and (c) its corresponding amplitude as defined in Eq. (A1). 
total low-frequency amplitude corresponding to all pairs of adjacent eigenvalues. Such curves demonstrate the resonant nature of the dynamical site-occupation as a function of the gate voltage. Figure 10(a) also shows that the low-frequency charge excitation peaks for intermediate gate voltages (for this case $V_{g} \sim 2.5 \gamma$ ) can be related to the observed enhanced low-frequency coupling between the itinerant and localized spins for the intermediate $V_{g}$ range. Interestingly, the two $A\left(V_{g}\right)$ curves coincide for very low and very high voltages, showing that in this case the entire dynamics is practically due to just one mode (marked in black in Fig. 9). In the intermediate regime it is clear that there is more than one low-frequency mode which has a significant amplitude, although the one marked in Fig. 9 gives the leading contribution.

For a particular pair of adjacent eigenstates there are a few instances where the adiabatic eigenvalues come closer together as a function of the gate voltage. In Figs. 10(b) and 10 (c) we analyze the dependence of the frequency and the amplitude corresponding to the pair of eigenvalues just below and above the Fermi level in the ground state ( $i=15$ for the 30 -atom chain at half filling). We find that the dynamical charge amplitude on the last site peaks (as absolute value) every time the frequency passes through a minimum.
${ }^{1}$ Spintronics, Vol. 82 of Semiconductors and Semimetals, edited by T. Dietl, D. D. Awschalom, M. Kaminska, and H. Ohno (Elsevier, Amsterdam, 2008).

${ }^{2}$ Spin Dynamics in Confined Magnetic Structures I, Topics in Applied Physics, edited by B. Hillebrands and K. Ounadjela (Springer, New York, 2002), Vol. 83.

${ }^{3}$ N. Baadji, M. Piacenza, T. Tugsuz, F. D. Sala, G. Maruccio, and S. Sanvito, Nature Mater. 8, 813 (2009).

${ }^{4}$ J. C. Slonczewski, J. Magn. Magn. Mater. 159, L1 (1996).

${ }^{5}$ E. B. Myers, D. C. Ralph, J. A. Katine, R. N. Louie, and R. A. Buhrman, Science 285, 867 (1999).

${ }^{6}$ S. Loth, K. von Bergmann, M. Ternes, A. F. Otte, C. P. Lutz, and A. J. Heinrich, Nature Phys. 6, 340 (2010).

${ }^{7}$ A. V. Kimel, A. Kirlyuk, P. A. Usachev, R. V. Pisarev, A. M. Balbashov, and Th. Rasing, Nature (London) 435, 655 (2005).

${ }^{8}$ A. Kirilyuk, A. V. Kimel, and Th. Rasing, Rev. Mod. Phys. 82, 2731 (2010).

${ }^{9}$ R. Hanson and D. D. Awschalom, Nature (London) 453, 1043 (2008).

${ }^{10}$ D. D. Awschalom, R. Epstein, and R. Hanson, Sci. Am. 297(4), 84 (2007).

${ }^{11}$ D. M. Toyli, C. D. Weis, G. D. Fuchs, T. Schenkel, and D. D. Awschalom, Nano Lett. 10, 3168 (2010).

${ }^{12}$ R. Jansen, J. Phys. D 36, R289 (2003).

${ }^{13}$ Y. Tserkovnyak, A. Brataas, and G. E. W. Bauer, Phys. Rev. Lett. 88, 117601 (2002).

${ }^{14}$ F. S. M. Guimarães, A. T. Costa, R. B. Muniz, and M. S. Ferreira, Phys. Rev. B 81, 233402 (2010).

${ }^{15}$ V. P. Antropov, M. I. Katsnelson, M. van Schilfgaarde, and B. N. Harmon, Phys. Rev. Lett. 75, 729 (1995); V. P. Antropov, M. I. Katsnelson, B. N. Harmon, M. van Schilfgaarde, and D. Kusnezov, Phys. Rev. B 54, 1019 (1996).

${ }^{16}$ E. Runge and E. K. U. Gross, Phys. Rev. Lett. 52, 997 (1984).

${ }^{17}$ K. L. Liu and S. H. Vosko, Can. J. Phys. 67, 1015 (1989).

${ }^{18}$ K. Capelle, G. Vignale, and B. L. Gyorffy, Phys. Rev. Lett. 87, 206403 (2001).

${ }^{19}$ A. P. Horsfield, D. R. Bowler, and A. J. Fisher, J. Phys. Condens. Matter 16, L65 (2004)

${ }^{20}$ J. Merino and O. Gunnarsson, Phys. Rev. B 69, 115404 (2004), and references therein.

${ }^{21}$ C. F. Hirjibehedin, C. P. Lutz, and A. J. Heinrich, Science 312, 1021 (2006); C. F. Hirjibehedin, C.-Y. Lin, A. F. Otte, M. Ternes, C. P. Lutz, B. A. Jones, and A. J. Heinrich, ibid. 317, 1199 (2007).

${ }^{22}$ A. Hurley, N. Baadji, and S. Sanvito, Phys. Rev. B 84, 035427 (2011).
${ }^{23}$ F. S. M. Guimarães, D. F. Kirwan, A. T. Costa, R. B. Muniz, D. L. Mills, and M. S. Ferreira, Phys. Rev. B 81, 153408 (2010).

${ }^{24}$ Time-Dependent Density Functional Theory, edited by M. A. L. Marques, C. A. Ullrich, F. Nogueira, A. Rubio, and K. Burke (Springer, Berlin, 2006).

${ }^{25} \mathrm{~K}$. Yosida, Theory of Magnetism (Springer-Verlag, Berlin, 1998), p. 228; J. Kondo, in Solid State Physics: Advances in Research and Applications, edited by F. Seitz, D. Turnbull, and H. Ehrenreich (Academic, New York, 1969), Vol. 23, p. 184.

${ }^{26} \mathrm{M}$. Stamenova and S. Sanvito, in The Oxford Handbook on Nanoscience and Technology (Oxford University Press, Oxford, 2010), Vol. 1, p. 169.

${ }^{27}$ M. Stamenova, T. N. Todorov, and S. Sanvito, Phys. Rev. B 77, 054439 (2008).

${ }^{28}$ W. Koshibae, N. Furukawa, and N. Nagaosa, Phys. Rev. Lett. 103, 266402 (2009).

${ }^{29}$ J. Thijssen, Computational Physics (Cambridge University Press, 2007), p. 473.

${ }^{30}$ W. H. Press, S. A. Teukolsky, W. T. Vetterling, and B. P. Flannery, Numerical Recipes: The Art of Scientific Computing (Cambridge University Press, 2007), p. 600.

${ }^{31}$ T. Giamarchi, Quantum Physics in One Dimension (Clarendon Press, Oxford, 2004), p. 12.

${ }^{32}$ J. des Cloizeaux and J. J. Pearson, Phys. Rev. 128, 2131 (1962); G. Muller, H. Thomas, H. Beck, and J. C. Bonner, Phys. Rev. B 24, 1429 (1981); O. Derzhko, in Condensed Matter Physics in the Prime of the 21st Century: Phenomena, Materials, Ideas, Methods, proceedings of the 43rd Karpacz Winter School of Theoretical Physics, Ladek Zdroj, Poland, edited by J. Jedrzejewski (World Scientific, 2008), p. 35.

${ }^{33}$ A. P. Horsfield, D. R. Bowler, A. J. Fisher, T. N. Todorov, and M. J. Montgomery, J. Phys. Condens. Matter 16, 3609 (2004).

${ }^{34}$ This region can also be reinterpreted as part of a different material so that the same model system also describes a one-dimensional trilayered heterostructure in which the two peripheral layers are identical.

${ }^{35}$ This effect can appear as a particular form of "heating" for the local spins, at the expense of electron gas, as within the time covered by the simulation $(\approx 10 \mathrm{ps})$ the energy transfer seems to be irreversible. It is well known, however, that the typical Ehrenfest molecular dynamics (EMD) suffers from the inability to account for Joule heating processes (Ref. 33) due to the lack of dynamical correlations between the electrons and the ions. Our simulations are limited to picosecond times and by no means imply a possible qualitative difference of the EMD and its spin counterpart. 